\title{
Effect of Electrolyte Concentration Difference on Hydrogen Pro- duction during PEM Electrolysis
}

\author{
Cheng-Wei Sun ${ }^{1}$ and Shu-San Hsiau,* \\ ${ }^{1}$ Dept. of Mechanical Engineering, National Central University, No.300, Jhongda Rd. Jhongli District, Taoyuan City 32001 , \\ Taiwan
}

\begin{abstract}
Proton exchange membrane (PEM) water electrolysis systems offer several advantages over traditional technologies including higher energy efficiency, higher production rates, and more compact design. In this study, all the experiments were performed with a self-designed PEM electrolyser operated at 1 atm and $25^{\circ} \mathrm{C}$. Two types of electrolyte were used: (i) potassium hydroxide $(\mathrm{KOH})$, and (ii) sulfuric acid $\left(\mathrm{H}_{2} \mathrm{SO}_{4}\right)$. In the experiments, the voltage, current, and time were measured. The concentration of the electrolyte significantly affected the electrolyser performance. Overall the best case was with $15 \mathrm{wt} \% \mathrm{H}_{2} \mathrm{SO}_{4}$ at the anode channel and $20 \mathrm{wt} \%$ at the cathode channel with. In addition, increasing the difference in concentration of the sulfuric acid had an effect on the diffusion. The diffusion flux became larger when the difference in concentration became larger, increasing electrolyser efficiency without the addition of extra energy.
\end{abstract}

Keywords : Proton exchange membrane electrolyser, Electrolysis, Electrolyte, Diffusion, Difference in concentration

Received : 17 January 2018, Accepted : 2 April 2018

\section{Introduction}

Hydrogen is an extremely abundant element on Earth, but its chemical properties mean that it cannot be stored in its standard state. It exists in states with a lower energy level like in water molecules and hydrocarbons. In recent years, the use of hydrogen as an energy carrier has become one possible solution for energy and ecological problems. Water electrolysis is a good way to produce hydrogen and techniques for extracting hydrogen by means of the application of electricity through water electrolysis have matured. Nowadays, studies of hydrogen production based on thermal dynamics and heat translation have led to the development of an operating model and suitable parameters for alkaline electrolysis. The voltage, operating temperature, and the amount of hydrogen produced [1] by electrolysis can be predicted from the temperature, pressure, and the

*E-mail address: sshsiau@cc.ncu.edu.tw

DOI: https://doi.org/10.5229/JECST.2018.9.2.99 relationship between the reactant and enthalpy [2]. The important parameters that affect hydrogen production include the reacting voltage, the electrolysis temperature (between $25^{\circ} \mathrm{C}-250^{\circ} \mathrm{C}$ ) and the operating pressure (between $1 \mathrm{~atm}-100 \mathrm{~atm}$ ) [3]. In addition to the derivation of the aforementioned model, the photoelectrochemical hydrogen production model is also used for the prediction of the relationship between the voltage and current density at low temperature, achieved by a combination of solar energy and proton exchange membrane (PEM) electrolysis [4]. This could be useful as a reference for the design of a method of hydrogen production by the application of solar energy.

Intensive efforts for the development of fuel cells, especially those based on the proton exchange membrane, where pure hydrogen is needed, have stimulated electrolyser evolution for future applications in the production of hydrogen energy and technology. The improvement of the electrolyser focuses on the design of the inner structure. For example, an appropriate catalyst can promote hydrolysis at the anode 
and reunion with hydrogen at the cathode. Recently, a new type of catalyst has been developed, which can operate reversibly at high efficiency [5]. The design and choice of material for the construction of a 2 cell stacked hydrogen production reactor allow for a reaction area of up to $100 \mathrm{~cm}^{2}$. Corrosion on the electrode surface and bad contacts during conduction affect the efficiency of the electrolyser, but heat generated by ohm impedance causes a heating reaction at the interface during the electrolysis process, which could promote the occurrence of electrochemical reactions to a certain degree [6]. The addition of even more could make the whole electrolyser spin at a constant high velocity. The centrifugal force would lower the overpotential and improve efficiency, thus the improvement of efficiency due to the spinning of the whole electrolyser could make up for the energy loss [7]. However, there has been relatively little research articles aimed at reducing energy loss. Increasing the operating temperature from $30^{\circ} \mathrm{C}$ to $90^{\circ} \mathrm{C}$ could increase the efficiency of hydrogen production [8], while increasing the operating pressure to $70 \mathrm{bar}$ and considering the ohm impedance effect on the electrolyser is found to increase the pressure which also increases the efficiency of hydrogen production in both experiment and theory [9]. Furthermore, there have been some improvements obtained by finding the optimal parameters, conditions for performance operation and energy efficiency for the electrolyser. Experiments have been carried out by using heated water cycles and electrode currents with two acid electrolytes, hydrogen bromide and sulfuric acid [10], or by using IrxRuy $\mathrm{TazO}_{2}$ electrodes, and changing the ratio between $\mathrm{Ta}$ and $\mathrm{Ru}$ [11].

Some practical considerations on the electrolyzers' development indicate that alkaline water electrolysis is a viable method for hydrogen production [12]. In a PEM electrolyser, the kind of exchange membrane used is very important. The application of the cation exchange membrane or anion exchange membrane causes a pressure drop due to the change in anion and cation transport which could affect the $\mathrm{PH}$ gradient. The pressure drop is lower in the anion exchange membrane electrolyser [13]. In the experiments undertaken in this study, four kinds of exchange membrane electrolysers are used with a cation exchange membrane (CEM), anion exchange membrane (AEM), bipolar mem- brane (BM), or charge mosaic membrane (CMM). It was found that when the electrolyte was sodium hydroxide, a higher current density was achieved with the AEM than the CEM while the BM had the lowest. The current density is proportional to the hydrogen generation. The total number of ions passing through the membrane is the greatest for the CEM followed by the AEM, with the CMM having the least [14]. The efficiency of the exchange membrane is affected by the impedance and the release of hydrogen between the reacting electrolytes. The change in the concentration of hydrogen after the electrolyte reaction causes a change in $\mathrm{PH}$ value and increases the impedance of the solution, which in turn causes a polarization in the concentration which has an effect on the efficiency of the electrolysis $[15,16]$. It has been found that the design of the electrolyser affects the aggregation of the hydrogen released. The horizontal or vertical setting of the board electrodes in sulfuric acid and potassium hydroxide electrolytes causes an obvious difference in the ohm impedance. Potassium hydroxide electrolysis produces denser bubbles than does sulfuric acid electrolysis, which means that the impedance is 2.5 times larger than with vertical setting of the electrodes [17]. Furthermore, bubble size, the rate of bubble rising, the number of bubbles produced, and other such parameters are considered in the calculation and affect the placement of the electrode $[18,19]$. The rate of hydrogen production increases with increase in concentration as an electrolyte. The input pressure, temperature and so on will affect the efficiency of electrolysis in all designs, but the electrolyte has the most direct effect on the ion concentration and conductivity of the solution during the electrolysis process, even changing the chemical reaction [20]. Thus, changing the electrolyte is the most efficient and fastest way to improve electrolysis.

The purpose of this study is to demonstrate the application at the present stage of the most advantageous PEM electrolyser; see Fig. 1 (a). Different concentrations of electrolyte are supplied to the cathode and anode leading to the occurrence of ion diffusion. We strive to improve the efficiency of PEM electrolysis in order to lower the cost of hydrogen production. The production of hydrogen from an efficient water electrolysis process could be combined with renewable energy applications. 


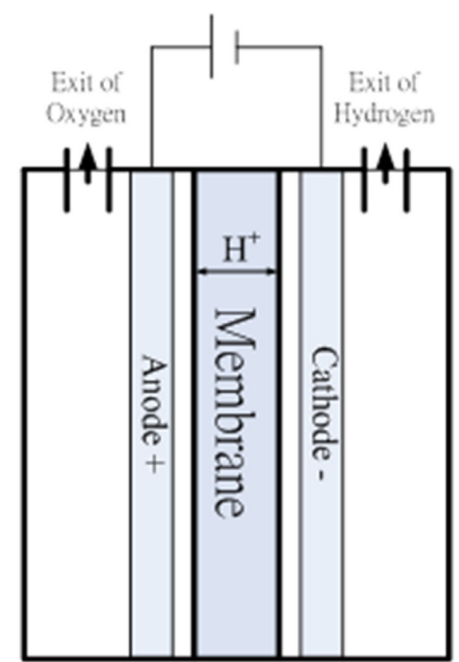

(a)

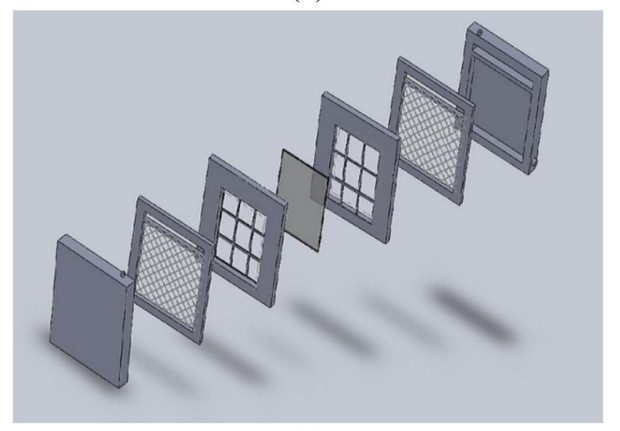

(b)

Fig. 1. Schematic drawing of a PEM electrolyser: (a) the structure of the electrolyser, and (b) the multi-layer design of the PEM electrolyser.

\section{Experimental}

\subsection{Materials and Methods}

\subsubsection{Electrolysis of Water}

Electrolysis of water is the decomposition of water $\left(\mathrm{H}_{2} \mathrm{O}\right)$ into oxygen $\left(\mathrm{O}_{2}\right)$ and hydrogen gas $\left(\mathrm{H}_{2}\right)$ due to an electric current being passed through the water. The parameters studied include the pressure, temperature and concentration of the electrolyte. In this study, the effects of differences in electrolyte concentration in a PEM electrolyser are discussed. Discussion of electrolysis is divided into two parts, because there are two kinds of electrolyte, acidic and alkaline. The acidic electrolytic chemical equations are as follows:
Anode: $2 \mathrm{H}_{2} \mathrm{O} \rightarrow \mathrm{O}_{2}+4 \mathrm{H}^{+}+4 e^{-}$,

Cathode: $4 H^{+}+4 e^{-} \rightarrow 2 \mathrm{H}_{2}$.

In alkaline electrolytes, the hydroxyl ions transmit electrons. The alkaline electrolytic chemical equations are as follows:

Anode: $4 \mathrm{OH}^{-} \rightarrow \mathrm{O}_{2}+2 \mathrm{H}_{2} \mathrm{O}+4 e^{-}$

Cathode: $2 \mathrm{H}_{2} \mathrm{O}+2 e^{-} \rightarrow \mathrm{H}_{2}+2 \mathrm{OH}^{-}$.

Combining either half reaction pair yields the same overall decomposition of water into oxygen and hydrogen:

$$
\text { Overall reaction: } 2 \mathrm{H}_{2} \mathrm{O} \rightarrow 2 \mathrm{H}_{2}+\mathrm{O}_{2} \text {. }
$$

The number of hydrogen molecules produced is thus twice the number of oxygen molecules. Assuming equal temperature and pressure for both gases, the produced hydrogen gas has therefore twice the volume of the produced oxygen gas. The number of electrons pushed through the water is twice the number of generated hydrogen molecules and four times the number of generated oxygen molecules.

\subsubsection{Electrolyser Design}

An electrolyte solution is formed by dissolving substances in a solvent. When electrical conductivity occurs in the solution, this substance is called an electrolyte and the solution is called an electrolyte solution. From as early as 1869 to 1880 , the electrical conductivity of the electrolyte solution has been measured and studied. Two pieces of the same electrode material are placed parallel in an electrolyte solution to produce an electric current which has a voltage. The electrolyser can be designed based on Ohm's Law, formulated as in the following equation:

$$
I=\kappa \frac{A}{x} \Delta \Phi,
$$

where $I$ is the current (A), $A$ is the surface area of the electrode $\left(\mathrm{cm}^{2}\right), x$ is the distance between the electrodes $(\mathrm{cm}), \Delta \Phi$ is the difference in the solution potential between the electrodes $(\mathrm{V})$, and $\kappa$ is the ratio constant. $\kappa$ can also be called the conductivity and expresses the characteristics of the current passing easily through in the electrolyte solution. When 
the distance between cathode and the anode is smaller, the current due to electrolysis is larger, given the same electrode, electrolyte solution, and potential. Therefore, electrodes with a large surface area and a small distance between them become part of the overall design of the electrolyser.

\subsubsection{Diffusion Effect}

Diffusion is one of several transport phenomena that occur in nature. A distinguishing feature of diffusion is that it results in mixing or mass transport, without requiring bulk motion. Thus, diffusion should not be confused with convection, or advection, which are other transport mechanisms that utilize bulk motion to move particles from one place to another.

There are two ways to introduce the notion of diffusion: either by taking a phenomenological approach, starting with Fick's laws of diffusion and their mathematical consequences, or a physical and atomistic one, consideration of the random walk of the diffused particles. In the phenomenological approach, according to Fick's laws, the diffusion flux is proportional to the negative gradient of concentrations. It goes from regions of higher concentration to regions of lower concentration. Various generalizations of Fick's laws have been developed within the framework of thermodynamics and non-equilibrium thermodynamics.

Fick's first law relates the diffusive flux to the concentration under the assumption of a steady state. It postulates that the flux goes from regions of high concentration to regions of low concentration, with a magnitude that is proportional to the concentration gradient, or in simplistic terms, the concept that a solute will move from a region of high concentration to a region of low concentration across a concentration gradient. For a one-dimensional case, the law is presented as:

$$
J=-D \frac{\partial \phi}{\partial x},
$$

where $J$ is the "diffusion flux", $J$ measures the amount of substance that will flow through a small area during a small time interval $\left(\mathrm{mol} / \mathrm{m}^{2} \mathrm{~s}\right), D$ is the diffusion coefficient or diffusivity in dimensions $\left(\mathrm{m}^{2} / \mathrm{s}\right), \phi$ is the concentration in dimensions $\left(\mathrm{mol} / \mathrm{m}^{3}\right)$, and $x$ is the position $(\mathrm{m})$. The negative sign indicates that $J$ moves in the opposite direction with the gradient.

\subsection{Experiments}

Currently, the operational mode of all types of fuel cell is the opposite of the decomposition of water. The efficiency of electrolysis in a PEM fuel cell can reach $50 \%$ to $95 \%$. The resultant material is separated from the cathode and the anode. And the purity of the hydrogen produced can be as high as $97 \%$. In this study, a PEM electrolyser is used.

\subsubsection{Experimental Devices}

The devices can be divided into two types: the PEM electrolyser and the measurement instruments. The PEM electrolyser used in the experiments was designed and developed by ourselves, following equation (6) and includes a cathode and an anode with a film that separates the gas. Gases are collected separately. The design is shown in Fig. 1 (b). The system used in this study comprises the following components:

Reactor: This is the space used to store the electrolyte. The cathode side and the anode side have a capacity of $250 \mathrm{~mL}$ each. There is an opening at the top of each side.

Area of reaction: Mesh $3.5 \mathrm{~mm} \times 6.0 \mathrm{~mm}$, Diameter $1.0 \mathrm{~mm} \times 1.0 \mathrm{~mm}$.

Electrode: This is placed between the reactor and the membrane. In the experiments conducted for this study, a $2 \mu \mathrm{m}$ thick surface platinum coating on a titanium mesh is used for the electrodes [21].

Membrane: DuPont's film 'Nafion ${ }^{\circledR} 117$ ' is used for the experiments.

Combination multi-chip design: This is used because it not only makes it easy to change the membrane and electrode but also connect the multi-reactor.

\subsubsection{Hydrogen Production}

In this study, the main parameter being varied is the electrolyte concentration. Acidic and alkaline solutions are used for the electrolytes. The acidic electrolyte is comprised of a $5 \mathrm{wt} \%-20 \mathrm{wt} \%$ aqueous solution of sulfuric acid. The alkaline electrolyte consists of a $10 \mathrm{wt} \%-40 \mathrm{wt} \%$ aqueous solution of potassium hydroxide. The experiments were conducted in the standard state with a constant pressure of $1 \mathrm{~atm}$, constant temperature of $25^{\circ} \mathrm{C}$ and fixed voltage of 2 volts. The relationship between the current and time was measured. The voltage was set between 0-3 volts, and the corresponding current value measured. The experimental parameters are shown in Table 1. 
Table 1. The experimental parameters used in this study.

\begin{tabular}{|c|c|c|c|c|c|c|c|c|c|c|}
\hline \multirow{2}{*}{$\begin{array}{c}\text { Pressure } \\
(\mathrm{atm})\end{array}$} & \multirow{2}{*}{ Membrane } & \multirow{2}{*}{$\begin{array}{c}\text { Reaction } \\
\text { Area } \\
\left(\mathrm{cm}^{2}\right)\end{array}$} & \multicolumn{2}{|c|}{ Electrode } & \multicolumn{2}{|c|}{$\begin{array}{l}\text { Concentration } \\
\text { of } \mathrm{KOH}\end{array}$} & \multicolumn{2}{|c|}{$\begin{array}{l}\text { Concentration } \\
\text { of } \mathrm{H}_{2} \mathrm{SO}_{4}\end{array}$} & \multicolumn{2}{|c|}{ Test voltage (V) } \\
\hline & & & Anode & Cathode & $\begin{array}{l}\text { Anode sink } \\
\text { (wt } \%)\end{array}$ & $\begin{array}{c}\text { Cathod } \\
\text { sink (wt } \%)\end{array}$ & $\begin{array}{l}\text { Anode sink } \\
(w t \%)\end{array}$ & $\begin{array}{c}\text { Cathod } \\
\operatorname{sink}(w t \%)\end{array}$ & I-V curve & I-t curve \\
\hline 1 & $\begin{array}{c}\text { Nafion }{ }^{\circledR} \\
117\end{array}$ & 140.8 & $\begin{array}{c}\text { Platinum } \\
\text { plating on } \\
\text { Titanium }\end{array}$ & $\begin{array}{c}\text { Platinum } \\
\text { plating on } \\
\text { Titanium }\end{array}$ & $\begin{array}{c}10,15,20 \\
25,30 \\
35,40\end{array}$ & $\begin{array}{l}25,30 \\
35,40\end{array}$ & $\begin{array}{l}5,10 \\
15,20\end{array}$ & $\begin{array}{l}5,10 \\
15,20\end{array}$ & $0-3$ & 2 \\
\hline
\end{tabular}

Note: Area of reaction: Mesh $3.5 \mathrm{~mm} \times 6.0 \mathrm{~mm}$, Diameter $1.0 \mathrm{~mm} \times 1.0 \mathrm{~mm}$

The experiment was divided into three stages, the combination of the electrolyser, activation of the membrane, and measurement of the voltage-current. The steps are described in detail below.

Combination of the electrolyser: An anti-leak gasket (O-Ring) was placed between the layers to prevent spillage. The distribution of torque for locked nuts should be noted [22].

Activation of the membrane: Activation of the membrane was carried out in preparation for the experiments. Activation during the experiments is needed to prevent breakage and bending caused by increased resistance [23]. Before the experiment, the exchange membrane must be activated according to the specification of the USFCC [24].

Measurement of the voltage-current: After activation the electrolyte is poured into the electrolyser and the measurement of voltage and current measurement started.

\section{Results and Discussion}

The experiments were carried out with varying concentrations of electrolyte solutions for the cathode and anode. A potentiostat was applied as the power supply, connecting the cathode and the anode as the negative and positive sides, respectively. All data were recorded by a computer and the current and electrolysis time controlled. The controlled variables were the electrolysis voltage, concentration of electrolyte solution, and electrolysis time. Observations and the results of the experiments helped to understand the effect of each parameter on the production of hydrogen from water electrolysis, and to find the optimal parameters for efficient hydrolysis. The electrolytes used in the experiment were sulfuric acid and potassium hydroxide. Different concentrations of electrolyte solution were prepared. The concentration of the hydrogen and hydroxyl ions varied, as did the current due to different impedances. In the experiments, the change of concentration due to polarization is defined as the Ohm's polarization. The impedance caused by differences in the electrolyser design might increase due to bubble production, or might differ due to changes of current or voltage.

\subsection{Effects of Hydrogen ions}

For the sulfuric acid electrolyte, the voltage ranged from $0-3$ volts, and the concentrations of the cathode of the electrolyser were $5 \mathrm{wt} \%, 10 \mathrm{wt} \%, 15 \mathrm{wt} \%$, and $20 \mathrm{wt} \%$. In Fig. 2, the horizontal axis is voltage and the vertical axis is the current. The aqueous sulfuric acid concentration in the cathode side was fixed. When the concentration of the sulfuric acid aqueous solution on the cathode side is fixed at $5 \mathrm{wt} \%$, the response current increases with the increase of voltage. When the gap of the concentration between the cathode side and the anode side is larger, the response current rises more, especially at $5 \mathrm{wt} \%$ on the cathode side, and $15 \mathrm{wt} \%$ or $20 \mathrm{wt} \%$ on the anode side. In all cases, hydrogen was produced at 1.4 volts. When the concentration of the electrolyte on the anode side is $5 \mathrm{wt} \%$ or $10 \mathrm{wt} \%$, there is a slower current response phenomenon at the beginning, as shown in Fig. 2 (a), but when concentration in the anode side is $15 \mathrm{wt} \%$, this situation does not occur. This may be affected by the bubbles. After the start of the electrolysis, the electrolysis inside the ohm impedance rises as bubbles continue to accumulate, resulting in a decrease in the efficiency of the electrolysis.

Fig. 2 (b), (c), (d) show the increase in the response current with the increase of voltage. When the gap of the concentration between the cathode side and the anode side is larger, the response current rises more, but a less obvious phenomenon is shown when the concentration on the cathode side is fixed at $20 \mathrm{wt} \%$. 
Electrolysis efficiency cannot be improved, because the concentration at the cathode side is too high. Hydrogen ions produced on the anode side cannot diffuse to the cathode side.

\subsection{Effects of Hydroxide ions}

In Fig. 3, for the aqueous solution of potassium hydroxide electrolyte, the voltage ranged from 0-3 volts, and the concentrations of the cathode of the electrolyser were $25 \mathrm{wt} \%, 30 \mathrm{wt} \%, 35 \mathrm{wt} \%$, and 40 wt $\%$. Fig. 3 (a) shows the concentration of potassium hydroxide fixed cathode at $25 \mathrm{wt} \%$, the response current was not due to the voltage increases significantly. Initial stage of electrolysis, after rise, the response current had a phenomenon slowdown and enhance again. This may be due to the impact of hydrogen and oxygen bubbles. When the bubbles accumulated to a certain extent, ohm impedance rise, resulting in decreased efficiency of electrolysis. But the greater concentration on the anode side, the concentration of the electrolyte solution increases, so that the concentration polarization decreases. The hydrogen produced by electrolysis voltage start in advance to around 1.4 volts.

In Fig. 3 (b), (c), (d), no matter how much the concentration of aqueous hydrogen hydroxide, it cannot enhance the efficiency of the electrolysis. This phenomenon should be hydroxide ion cannot through proton exchange membrane. Therefore, the concentration difference of hydroxide solution on cathode and the anode side did not effectively help the overall electrolysis. When the concentration of aqueous hydrogen hydroxide on anode side up to $35 \mathrm{wt} \%$, the electrolysis efficiency decreased. Because the con-
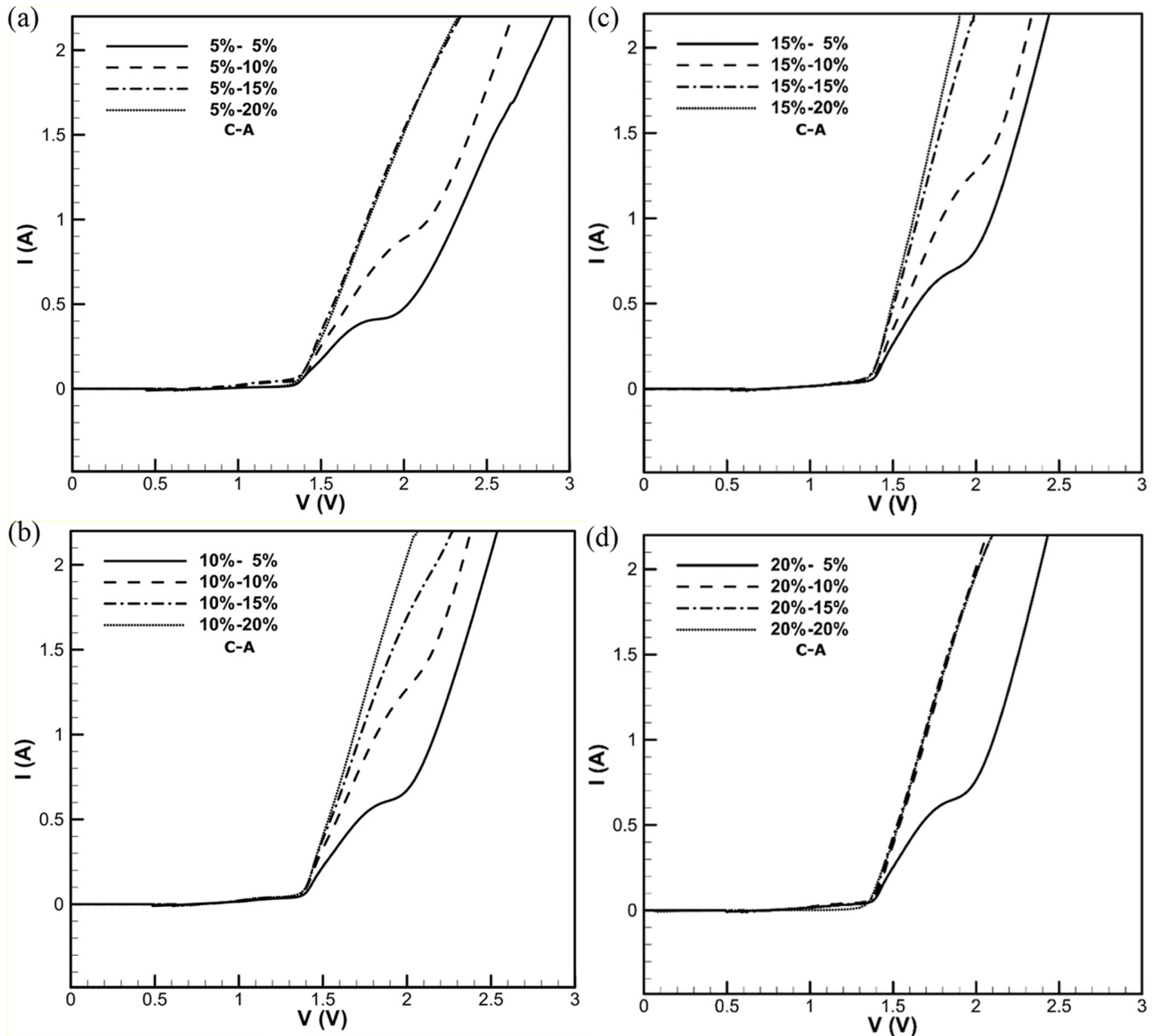

Fig. 2. I-V curves for the voltage range between 0-3 volts. The concentration of the aqueous sulfuric acid on the cathode side is fixed at (a) $5 \mathrm{wt} \%$, (b) $10 \mathrm{wt} \%$, (c) $15 \mathrm{wt} \%$, and (d) $20 \mathrm{wt} \%$. 
centration of potassium hydroxide solution is too high, leaving the liquid conductivity decreased, resulting in electrolysis efficiency slowed.

\subsection{Effects of Impedance}

Bubbles were one of the cause of the ohm impedance. Figs. 2 and 3 show the curve of response current with a concussion phenomenon, after the start of electrolysis. This decline in the performance was speculated to be caused by the bubbles. Micro-bubbles produced by electrolysis of occupied space, they obstructed the path of the ions in the electrolyte. If the proportion of air bubbles in the electrolytic solution increases, the large number of bubbles were uniformly mixed in the liquid. Ion transport conduction path was blocked, and caused the impedance between the electrodes and the proton exchange membrane increased [17]. Electrolysis reaction, the ion path is very important, otherwise it would endanger the electrolyser performance and cause polarization of the electrode surface.

\subsection{Transport Phenomena}

For a fixed concentration of sulfuric acid at the anode, there was an increase in the current as the concentration of the sulfuric acid solution on the cathode side was increased. When the concentration at the anode increased from $5 \mathrm{wt} \%$ to $20 \mathrm{wt} \%$, there was also an increase in the response current. The difference in concentration is shown in Fig. 4, where the horizontal axis shows the difference in sulfuric acid concentration between the cathode and the anode side. The vertical axis shows the current at steady state. In the figure we can see a significant difference in the concentration of
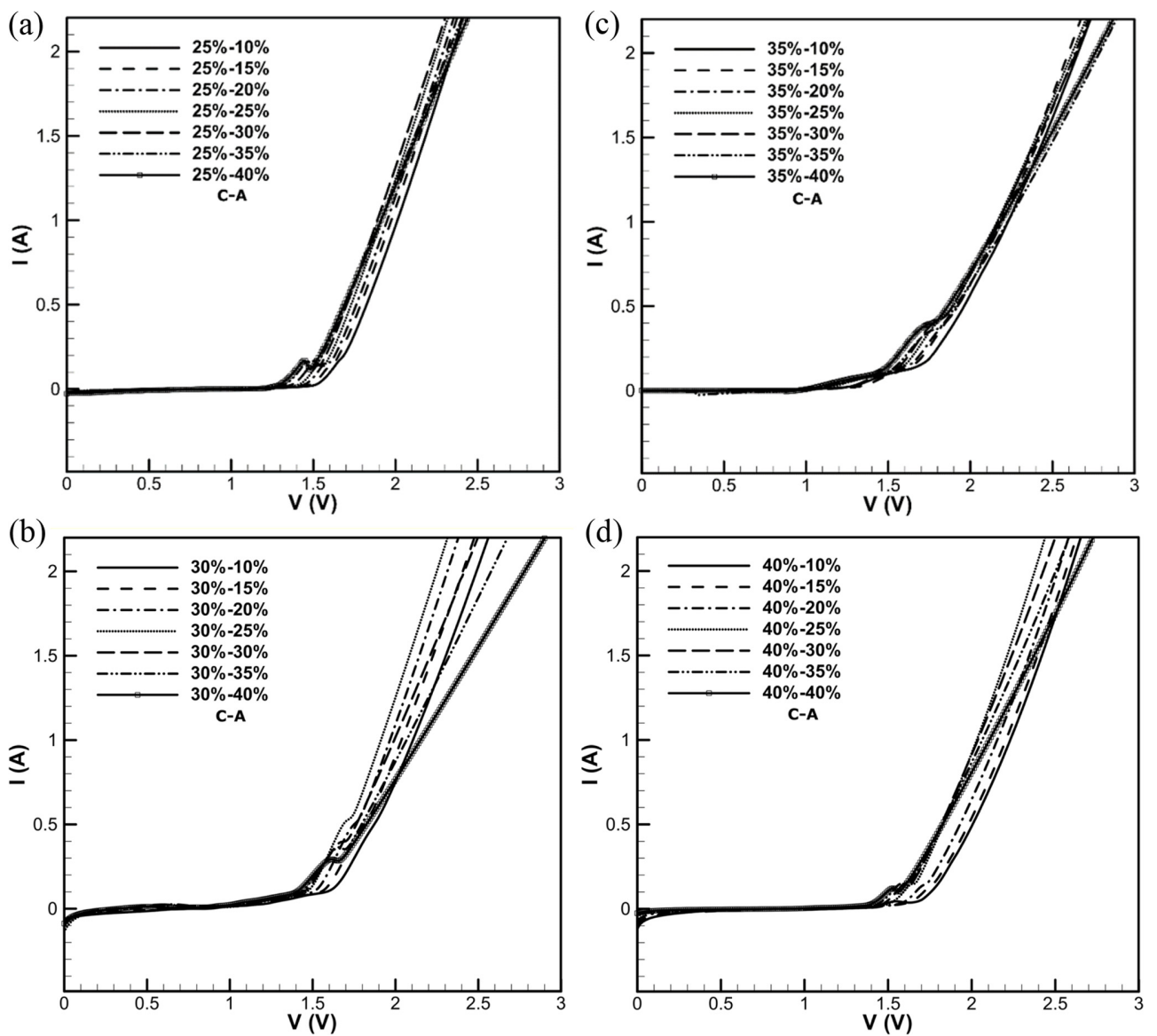

Fig. 3. I-V curves for a voltage range between $0-3$ volts. The concentration of the aqueous solution of potassium hydroxide on the cathode side is fixed at (a) $25 \mathrm{wt} \%$, (b) $30 \mathrm{wt} \%$, (c) $35 \mathrm{wt} \%$, and (d) $40 \mathrm{wt} \%$. 


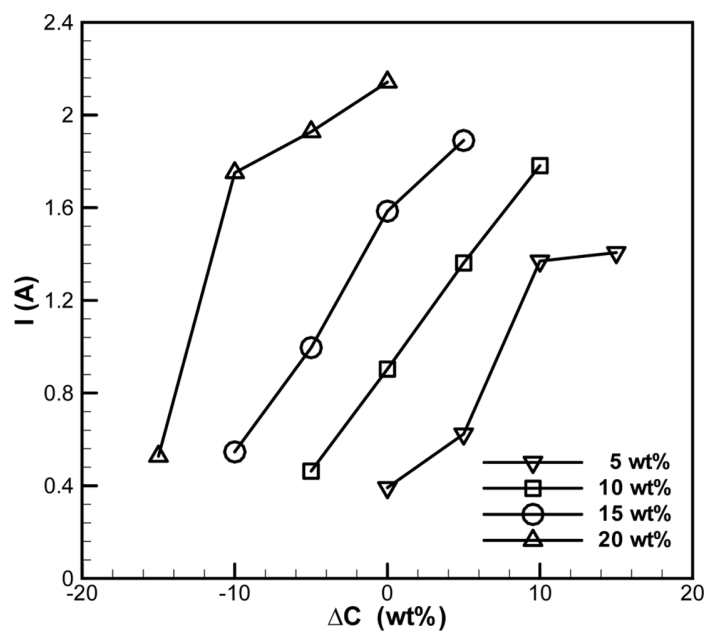

Fig. 4. For a fixed concentration of sulfuric acid solution on the cathode side, the relationship of the response current to the difference in concentration between the anode and cathode sides.

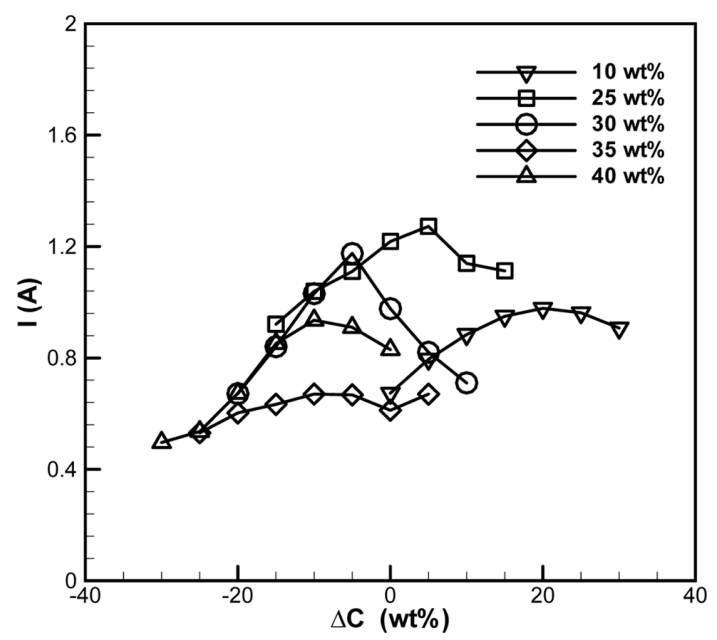

Fig. 5. For a fixed concentration of potassium hydroxide solution on the anode side, the relationship of the response current to the difference in concentration between the cathode and anode sides.

the sulfuric acid between two electrodes, which has a great effect on the response current.

With a fixed concentration of potassium hydroxide on the anode side, there was an increase in the response current as the concentration of potassium hydroxide on the cathode side increased, as shown in
Fig. 5. And the vertical axis shows the current at steady state. However, after the concentration increased from $5 \mathrm{wt} \%$ to $30 \mathrm{wt} \%$, the response current reached a maximum. When the concentration at the anode increased from $5 \mathrm{wt} \%$ to $20 \mathrm{wt} \%$, the response current also increased. There is an apparent difference in concentration. However, increasing the difference does not lead to a diffusion effect. This is because the proton exchange membrane prevented the penetration of hydroxide ions. Thus, the difference in concentration between the two electrodes did not cause any effect.

The electrolysis current can be viewed as energy, to be discussed in relation to the concentration. When the proton exchange membrane is wet, hydrogen ions bond easily with the hydrophilic side chain and move freely with water between the hydrophobic chains. This property is what gives the membrane the same chemical property as the electrolyte. In the sulfuric acid experiments, sulfuric acid and the proton exchange membrane played the role of the electrolyte in the transmission of ions. The concept of impedance is considered because of the energy carriers provided from the electrolysis current and electrolyte provided from the proton exchange membrane. There is an increase in electrolysis efficiency when the electrolyte concentration of the acid is higher at the cathode than at the anode, and the diffusion effect due to the difference in concentration applies. However, it is the hydroxyl ions in the potassium hydroxide that play the role of electrolyte conduction ions, because the hydroxyl ions cannot be transmitted through the proton exchange membrane. Thus, whatever the concentration of the base electrolyte at the cathode or the acid electrolyte at the anode, the difference in concentration cannot cause a diffusion effect that increases the efficiency of electrolysis. However, appropriately increasing the concentration still made electrolysis happen by increasing the conductivity.

Fig. 6 shows that the response current ratio increases with the concentration ratio. The response current ratio and the concentration ratio equations are as follows:

$$
\begin{gathered}
C_{R}=\frac{C_{a}}{C_{c}} \\
I_{R}=\frac{I_{a}}{I_{c}}
\end{gathered}
$$




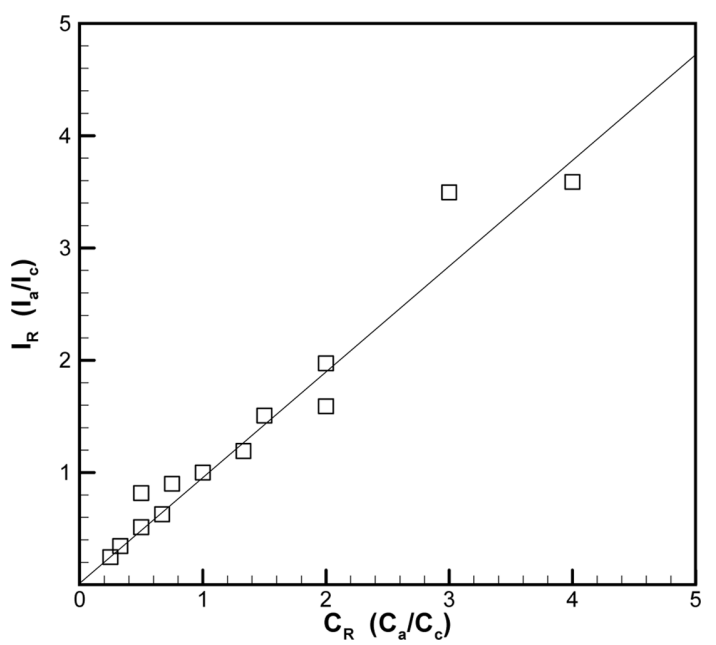

Fig. 6. The relationship between the concentration ratio and the response current ratio.

where $C_{R}$ is the concentration ratio, $C_{a}$ is the concentration of electrolyte at the anode side, $C_{c}$ is the concentration of electrolyte at the cathode side, $I_{R}$ is the response current ratio, $I_{c}$ is the response current for the corresponding concentration $C_{c}$, and $I_{a}$ is the response current for the corresponding concentration $C_{a}$.

When the concentration ratio $\left(C_{R}\right)$ is higher than 1 , the response current ratio $\left(I_{R}\right)$ also becomes higher than 1 . This means that when the concentration of the anode side is higher than the cathode side, the hydrogen ions passing through the exchange membrane tend to increase. So the diffusion flux becomes larger when the difference of concentration becomes higher, conforming to equation (7).

There is a prediction curve derived from the linear fit. It means the response current increases with the increase of the difference in concentration, and it is possible to estimate the response current in the case of different concentration differences.

\section{Conclusions}

In this study, two electrolyte solutions with different PHs were tested: one was a sulfuric acid solution, and the other was a potassium hydroxide solution. We obtained the following conclusions from the experiments:

The concentration of electrolytes was varied, and the effect on the electrolysis was obvious. In the stan- dard situation, there was an optimized parameter with different concentrations at the anode and cathode. The efficiency of electrolysis was better when the voltage was fixed at 2 volts, and the potassium hydroxide solution concentration was $25 \mathrm{wt} \%$ at the anode and $30 \mathrm{wt} \%$ at the cathode. The reason for this phenomenon was hydroxide ion cannot through proton exchange membrane. However, hydroxyl ions gradually saturated the anode, so the concentration at the anode was in excess of $25 \mathrm{wt} \%$, at which point the conductivity had a negative tendency.

When the sulfuric acid solution concentration was higher at the cathode than at the anode, the ion diffusion effect became significant, leading to the transmission of hydrogen ions through the proton exchange membrane at the cathode, which then moved to the anode to work continuously for electrolysis. The best case was with 15 $w t \% \mathrm{H}_{2} \mathrm{SO}_{4}$ at the anode channel and with $20 \mathrm{wt} \%$ at the cathode channel. In addition, increasing the difference in concentration of the sulfuric acid had an effect on the diffusion.

In the experiments, increasing the difference in concentration of the sulfuric acid had an effect on the diffusion. This fulfilled the purpose of this study, namely increasing electrolyser efficiency without the addition of energy.

\section{References}

[1] O. Ulleberg, Int. J. of Hydrogen Energy, 2003, 28(1), 21-33.

[2] K. Onda, T. Kyakuno, K. Hattori, and K. Ito, J. Power Sources, 2004, 132(1-2), 64-70.

[3] R. L. LeRoy, C. T. Bowen, and D. J. LeRoy, J. Electrochem. Soc., 1980, 127, 1954-1962.

[4] J. Nie, Y. Chen, R. F. Boehm, and S. Katukota, J. Heat Transfer, 2008, 130, 1-6.

[5] T. Ioroi, K. Yasuda, Z. Siroma, N. Fjuiwara, and Y. Miyazaki, J. Power Sources, 2001, 112(2), 583-587.

[6] P. Millet, F. Andolfatto, and R. Durand, Int. J. Hydrogen Energy, 1996, 21(2), 87-93.

[7] H. Cheng, K. Scott, and C. Ramshaw, J. Electrochem. Soc., 2002, 149(11), D172-D177.

[8] S. A. Grigoriev, V. I. Porembsky, and V. N. Fateev, Int. J. Hydrogen Energy, 2006, 31(2), 171-175.

[9] F. Marangio, M. Santarelli, and M. Cali, Int. J. Hydrogen Energy, 2009, 34(93), 1143-1158.

[10] P. Sivasubramanian, R. P. Ramasamy, F. J. Freire, C. E. Holland, and J. W. Weidner, Int. J. Hydrogen Energy, 2007, 32(4), 463-468.

[11] A. Marshall, B. Borresen, G. Hagen, M. Tsypkin, and R. Tunold, Energy, 2005, 32(4), 431-436. 
[12] Diogo M. F. Santos and César A. C. Sequeira, Quim. Nova, 2013, 36(8), 1176-1193.

[13] R. A. Rozendal, H. V. M. Hamelers, R. J. Molenkamp, and C. J. N. Buisman, Water Research, 2007, 41(9), 1984-1994.

[14] R. A. Rozendal, T. H. J. A. Sleutels, H. V. M. Hamelers, and C. J. N. Buisman, Water Science \& Technology, 2008, 57(11), 1757-1762.

[15] M. D. Merrill, B. E. Logan, J. Power Sources, 2009, 191(2), 203-208.

[16] M. Macka, P. Andersson, and P. R. Haddad, Anal. Chem., 1998, 70(4), 743-749.

[17] D. Kiuchi, H. Matsushima, Y. Fukunaka, and K. Kuribayashi, J. Electrochem. Soc., 2006, 153(8), E138E143.

[18] P. J. Sides, C. W. Tobias, J. Electrochem. Soc., 1980, $127,288-291$
[19] G. Kreysa, H. J. Kulps, J. Electrochem. Soc., 1981, 128(5), 979-984.

[20] Biswajit Mandal, Amalesh Sirkar, Parameswar De, and Sunil Baran Kuila, International Journal of Research in Engineering and Technology, 2016, 05, 8-12.

[21] Fumihiro Kodera, Yu Kuwahara, Akira Nakazawa, and Minoru Umeda, J. Power Sources, 2007, 172(2), 698703.

[22] C. Y. Wen, Y. S. Lin, and C. H. Lu, J. Power Sources, 2009, 192(2), 475-485.

[23] Z. Qi, A. Kaufman, J. Power Sources, 2002, 111(1), 181-184.

[24] US Fuel Cell Council, Single Cell Test Protocol, US Fuel Cell Council, 2006. 